\title{
Sistema automatizado para medición del coeficiente de fricción estática. Un dispositivo para actividades de docencia
}

Automated system for measuring the static friction coefficient. A device for learning activities

\author{
Jesús González-Laprea*1] ${ }^{*}$, Santiago Álvaro ${ }^{1}$ \\ ${ }^{1}$ Pontificia Universidad Católica del Ecuador, Facultad de Ciencias Exactas y Naturales, \\ Escuela de Ciencias Físicas y Matemáticas, Quito, Ecuador.
}

Recibida en 11 de Febrero, 2021. Revisado en 26 de Febrero, 2021. Aceptado en 01 de Marzo, 2021.

\begin{abstract}
Se presenta un dispositivo para la determinación del coeficiente de fricción estática diseñado para mejorar la experiencia en la toma de datos, sin sacrificar su contenido didáctico. El sistema, basado en la medición del ángulo crítico de deslizamiento para un objeto sobre un plano inclinado, utiliza un sensor de posición, eliminando el factor tiempo de reacción en la medida. Un microcontrolador ARDUINO UNO, permite la adquisición y registro de datos de posición y ángulo de inclinación del plano en forma simultánea, con lo que se determina el ángulo crítico. Adicionalmente se incluye un mecanismo de variación continua del ángulo del plano mediante una conexión mecánica no solidaria, reduciendo así los movimientos bruscos o la transmisión de vibraciones del motor a la superficie de medición. Con el sistema propuesto se obtienen coeficientes de fricción con un alto grado de reproducibilidad, proporcionando valores que no sobrepasan el $3 \%$ de error en las medidas. El sistema fue probado, usando igual metodología, con superficies de diferentes características, obteniéndose resultados satisfactorios. Como prueba de confiabilidad, se utilizó el equipo en un conjunto de experimentos para determinar la influencia de vibraciones mecánicas sobre los resultados.

Palabras clave: Coeficiente de fricción estática, plano inclinado, automatización con ARDUINO, prácticas de laboratorio de docencia.
\end{abstract}

A device for determination of static friction coefficient is presented, which has been designed to improve the data acquisition experience, without sacrificing its educational content. The system, based on the measurement of the critical sliding angle for an object on an inclined plane, it uses a position sensor, which removes the reaction time factor from the measurement. An ARDUINO UNO microcontroller allows for the acquisition and recording of the position and plane inclination angle simultaneously, this allows for determination of the critical sliding angle. Additionally, an automatic mechanism to continuously vary the plane angle by means of a non-solidary mechanical connection is included, thus reducing abrupt movements or the transmission of vibrations from the motor to the surface upon which the measurement is made. Using the proposed system, friction coefficients with high degree of reproducibility are obtained, with values that do not exceed a $3 \%$ rate of error for measurements. The system was tested, using the same methodology, on surfaces of different types, and satisfactory results were obtained. As a reliability test, the device was used in a set of experiments to determine the effect of mechanical vibrations on the results.

Keywords: Static friction coefficient, inclined plane, automation with ARDUINO, teaching laboratory practices.

\section{Introducción}

Ya que la Física es una ciencia que implica en igual medida tanto el trabajo teórico como el trabajo experimental, su enseñanza debe contener ambos componentes. Esta es la razón por la que la mayoría de los currículos de Física, a nivel mundial, incluyen cursos (o al menos sesiones) de prácticas de laboratorio; esto permite a los estudiantes entrar en contacto directo con los fenómenos estudiados en el salón de clases y aprender técnicas y metodologías de medición. Dentro de los cursos de Mecánica y en particular, incluido entre las actividades

\footnotetext{
* Correo electrónico: jegonzalezl@puce.edu.ec
}

de laboratorio, se encuentra normalmente el estudio de la fuerza de fricción [1,4.

A nivel instruccional, la fuerza de fricción normalmente es tratada al momento de presentar las leyes de Newton, y pasa a formar parte del conjunto de fuerzas que actúan sobre un cuerpo [3 5]. Específicamente, la fricción se plantea como una componente de la fuerza de contacto que surge al apoyar un cuerpo sobre una superficie o al poner dos cuerpos en unión. Esta fuerza tiene entonces dos componentes, una perpendicular a la superficie de contacto, denominada comúnmente como fuerza normal, y una componente paralela a la superficie, que es la denominada fuerza de Fricción. En su forma más simple, la existencia de la fuerza de fricción es 
atribuida a la interacción que se da a nivel microscópico entre las superficies en contacto, así, esta interacción es mediada o bien por efectos electrostáticos como fuerzas de Van Der Waals, o bien por el simple atascamiento entre las imperfecciones de ambas superficies, similar a lo que ocurriría, pero a una mucho menor escala, entre un engrane y una cremallera. Debido a lo anterior, mientras mayor sea la presión ejercida entre las superficies, mayor será el atrancamiento entre éstas y por lo tanto podría ser mayor la fuerza de fricción [6, [7].

El cómo actúa esta fuerza de fricción sobre un cuerpo depende en primera instancia de si existe movimiento relativo entre las dos superficies en contacto; si hay movimiento relativo se habla de fricción cinética o dinámica $\left(f_{k}\right)$, en caso contrario, se habla de fricción estática $\left(f_{s}\right)$. La fuerza de fricción cinética se define, en su forma más sencilla, como una fuerza uniforme dirigida a lo largo de la dirección de movimiento relativo entre las superficies y, en sentido contrario al movimiento relativo del cuerpo sobre el que actúa. Aun cuando esta fuerza es uniforme, sigue viéndose influenciada por la presión entre las superficies en contacto, de esta forma, la magnitud de esta fuerza va a estar dada por el producto entre la magnitud de la fuerza normal (debida a la otra superficie en contacto) y un factor denominado coeficiente de fricción cinética (dinámica), que engloba todos estos elementos de interacción microscópica entre las superficies 6-8.

La fuerza de fricción estática no es una fuerza uniforme; si una segunda fuerza actúa sobre el cuerpo y tiene una componente paralela a la superficie de contacto de éste, esta fuerza tiene que ser lo suficientemente grande como para lograr vencer las interacciones microscópicas entre las superficies, es decir, tiene que ser suficientemente fuerte para vencer las fuerzas Van Der Waals o para lograr destrabar las superficies, mientras no lo sea, no será capaz de hacer que el cuerpo comience a moverse. Desde el punto de vista macroscópico la fuerza de fricción estática se entiende como capaz de aumentar linealmente en magnitud, para oponerse a una fuerza antiparalela, hasta un punto máximo donde "se ve superada" y el cuerpo comienza a moverse. Una representación esquemática de esto puede ser vista en la Figura 1. tomada de un libro comúnmente utilizado para cursos de Física universitaria [9].

El punto máximo de magnitud de la fuerza de fricción estática, al igual que con la fricción cinética, depende de la presión entre las superficies, para el caso de la magnitud de la fuerza de fricción estática $\left(f_{s}\right)$ está dada por la ecuación (1):

$$
f_{s}=\mu_{s} \cdot|\vec{n}|
$$

donde $n$ es la magnitud de la fuerza normal sobre la superficie y $\mu_{s}$ es denominado coeficiente de fricción estática. Al igual que con el coeficiente de fricción cinética, $\mu_{s}$ es la representación macroscópica de las interacciones microscópicas entre las superficies y depende

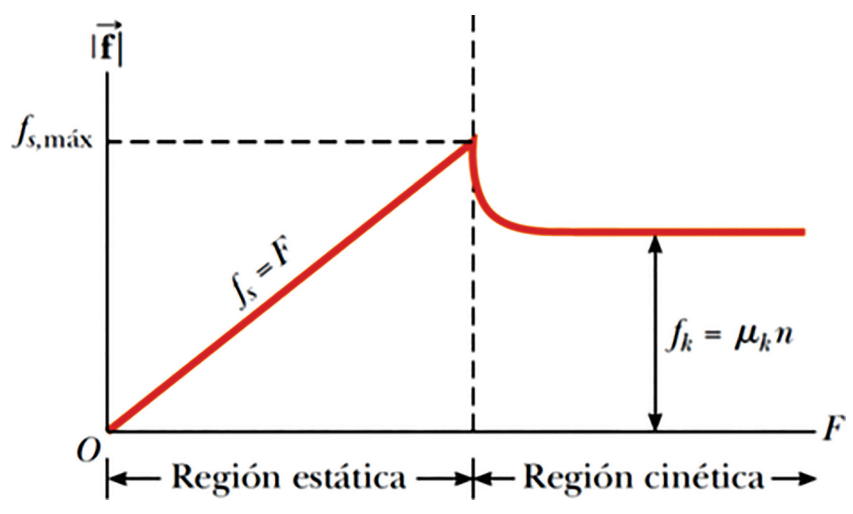

Figura 1: Magnitud de la fuerza de fricción en función de una fuerza aplicada de forma antiparalela. Se observan las regiones de comportamiento cinético y estático. Tomado de [9].

únicamente de las características de las superficies en contacto, no del tamaño del área en contacto [3, 4, 7, 10].

Una de las formas que se emplea tradicionalmente en laboratorios de docencia para determinar el coeficiente de fricción estática, consiste en colocar un cuerpo sobre un plano inclinado de ángulo ajustable, posteriormente el estudiante incrementa progresivamente el ángulo hasta el momento en que el cuerpo comienza a deslizar; el momento justo antes de deslizar, cuando se alcanza un ángulo crítico, es donde se cumple (1) [11. Realizando el diagrama de cuerpo libre sobre el móvil, justo en este instante, se observa que la única fuerza en contraposición a la fricción estática es la componente del peso paralela al plano inclinado, y como el sistema aún se mantiene en equilibrio, según la segunda ley de Newton se obtiene la ecuación (2):

$$
f_{s}-m g \sin \left(\alpha_{c}\right)=0
$$

donde $m$ será la masa del cuerpo, $g$ la magnitud de la aceleración de gravedad y $\alpha_{c}$ el ángulo de inclinación del plano justo antes que el cuerpo comience a deslizar (ángulo crítico). Para determinar el coeficiente de fricción estática a partir de las ecuaciones (1) y (2) es necesario conocer la magnitud de la fuerza normal $(n)$, La cual se obtiene al resolver el diagrama de cuerpo libre sobre la dirección perpendicular al plano, obteniéndose la ecuación (3):

$$
|\vec{n}|-m g \cos \left(\alpha_{c}\right)=0
$$

Finalmente, sustituyendo (2) y 3 en (1) se puede llegar a una expresión que relaciona $\mu_{s}$ con el ángulo crítico del plano:

$$
\mu_{S}=\tan \left(\alpha_{c}\right)
$$

Cuando se determina el coeficiente de fricción por esta vía, la persona que realiza la medida debe estar alerta al movimiento del cuerpo, reaccionar rápidamente para detener el plano y realizar la medida del ángulo, así 
mismo, el movimiento del plano debe ser los más fluido posible de forma que se eviten movimientos bruscos o vibraciones que hagan deslizar el cuerpo antes de alcanzar el ángulo crítico. Todo esto hace que, aunque la medida sea fácil de implementar en el laboratorio, se vuelva poco precisa y por tanto acaree errores de medida considerablemente grandes.

Una forma de reducir los efectos del experimentador y mejorar la reproducibilidad de la medida, puede ser mediante el uso de sensores que permitan minimizar el efecto del tiempo de reacción del operario. Hoy en día se tiene acceso a muchos sistemas de código abierto, de fácil y económica implementación, como las placas de microcontrolador ARDUINO, que funcionan en conjunto con una gran diversidad de sensores y controladores. Mediante este tipo de sistemas, se ha podido digitalizar el registro de datos y control de una cantidad importante de procedimientos experimentales, trayendo consigo un gran auge en su uso [4, 12 14. Este tipo de sistemas incluso han sido utilizados en dispositivos para la determinación de coeficientes de fricción cinética, con móviles desplazándose sobre superficies de inclinación fija, como el presentado por Sari en 2019 [4].

Basándonos en lo anterior, en este trabajo se propone implementar un sistema para la determinación del coeficiente de fricción estática entre dos superficies sólidas en contacto, que permita mejorar el grado de reproducibilidad de los resultados obtenidos pero manteniendo el aporte didáctico de soluciones similares. Adicionalmente, se espera que gracias a su bajo costo pueda ser implementado dentro del conjunto de actividades frecuentes en laboratorios de docencia en Física, orientados a Mecánica Newtoniana.

\section{Diseño y funcionamiento del dispositivo}

La parte mecánica del dispositivo consiste en una superficie de $(50,0 \pm 0,1) \mathrm{cm}$ de largo y $(7,0 \pm 0,1) \mathrm{cm}$ de ancho, construida a partir una plancha de acrílico de $(6 \pm 1) \mathrm{mm}$ de espesor y reforzada en los laterales con el objeto de mantener la estabilidad requerida para realizar los exprimentos. Este plano inclinado pivotea respecto a una superficie fija horizontal y es elevado en su otro extremo por medio de un cordel poco extensible. El cordel es enrollado en un carrete unido solidariamente a un motor de pasos (modelo 28BYJ-48, de 512 pasos por giro), que al suministrar un torque constante permite el aumento continuo y sin saltos en la inclinación del plano (Figura 2). El hecho de que la unión entre el motor de pasos y el plano inclinado sea a través de un elemento flexible como el cordel, permite minimizar la transmisión de las vibraciones (inherentes al motor) al plano.

El proceso para el registro de datos es llevado a cabo por medio de dos sensores conectados a una placa ARDUINO UNO: El primero es un sensor de posición por ultrasonido (HC-SR04), fijado en la parte inferior

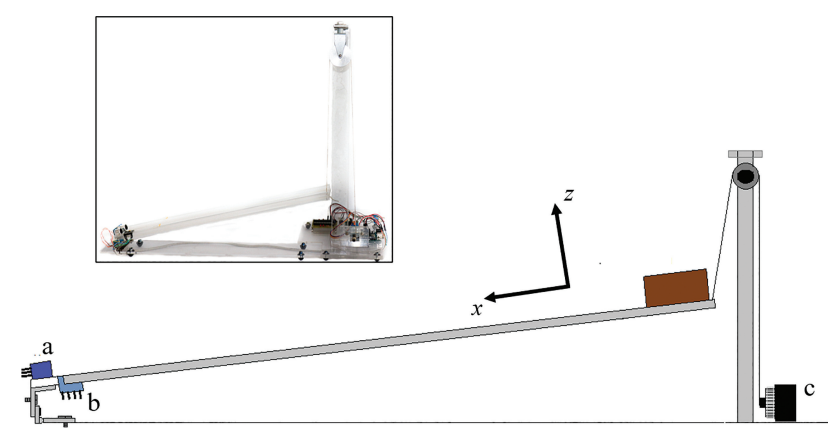

Figura 2: Representación esquemática del dispositivo utilizado. Sobre el plano de inclinado regulable se encuentran el sensor de posición (a) y el sensor de aceleración XYZ (b). La elevación del plano es llevada a cabo con un motor de pasos a través de un cordel inextensible (c). En el inserto se presenta una fotografía del montaje experimental.

del plano inclinado (Figura 2-a), con éste, se determina el momento justo en que el objeto en estudio comienza a desplazarse o, en otras palabras, vence la fricción estática y comienza su movimiento. El segundo sensor es un acelerómetro triaxial (ADXL345) que se mueve junto con el plano inclinado (Figura 2-b), esto permite tener información en tiempo real de las componentes de la aceleración de gravedad paralela al plano y perpendicular a éste. Considerando que todo el sistema se encuentra estático, se toma la dirección $-x-$ como la dirección a lo largo del plano y la dirección $-z-$ como la dirección perpendicular a éste, entonces el ángulo del plano respecto a la horizontal $(\alpha)$ estará dado por la ecuación (5):

$$
\alpha=\tan ^{-1}\left(\frac{a_{x}}{a_{z}}\right)
$$

donde $a_{x}$ y $a_{z}$ son las componentes de la gravedad a lo largo de los respectivos ejes. La Figura 3 muestra el esquema de conexión de los sensores a la placa ARDUINO UNO.

El motor que determina el movimiento del plano inclinado (Figura 2 c) es controlado por medio de un microcontrolador (16F877A) aislado de la placa ARDUINO UNO, esto se hizo para evitar los retrasos inherentes al procesamiento de datos que también realizaría la placa, logrando un movimiento mucho más fluido del motor. La instrucción para subir o bajar el plano (enrollar o desenrollar cordel) se da al motor de pasos por medio de la activación de un interruptor de 3 puntos, la Figura 4 muestra el esquema del circuito empleado para el control del motor de pasos.

Para determinar el coeficiente de fricción, la información proveniente de los dos sensores (posición y ángulo) es graficada simultáneamente en función de una base temporal arbitraria, el gráfico obtenido se puede observar en la Figura 5 . Como ambas curvas suceden en el mismo intervalo de tiempo basta con identificar en la que corresponde a la posición del cuerpo el punto donde 


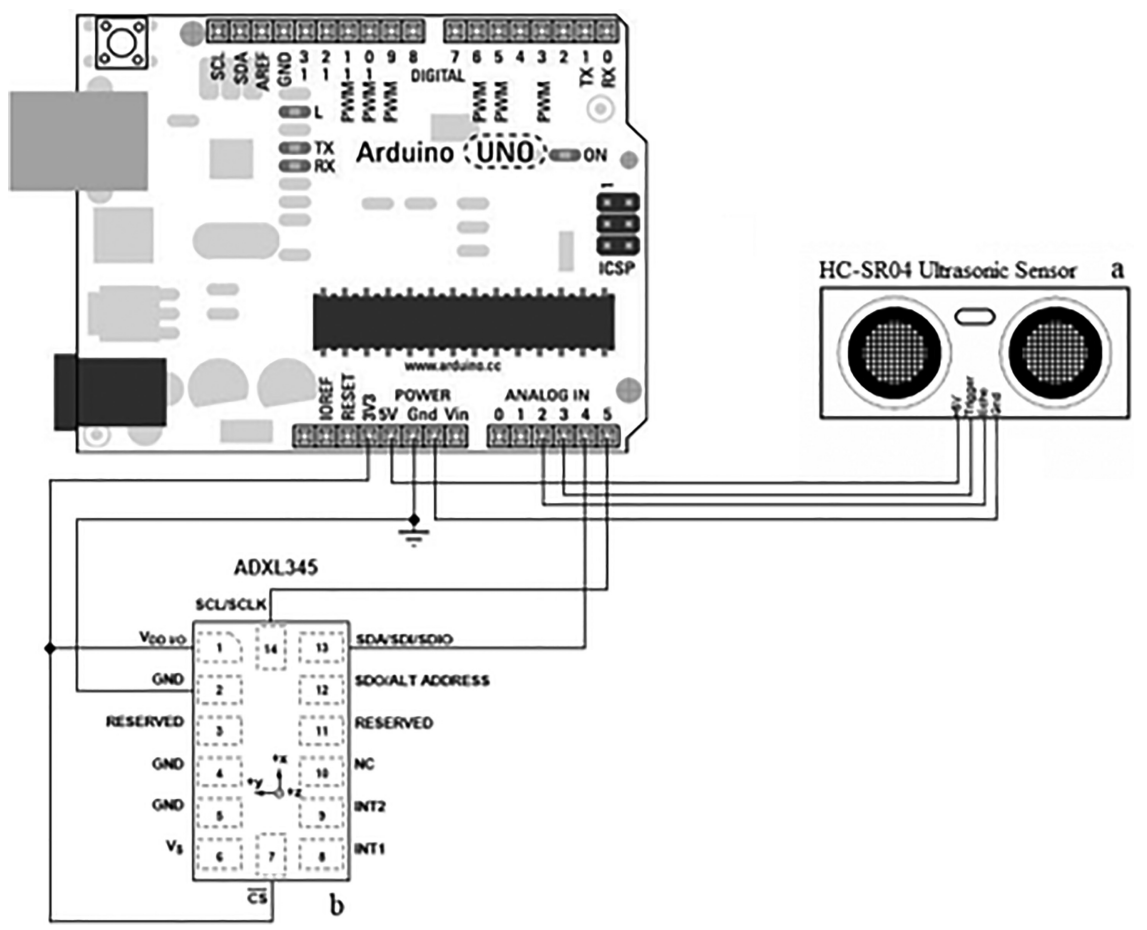

Figura 3: Conexión de los sensores a la placa ARDUINO UNO, (a) sensor de posición HC-SR04, (b) sensor de aceleración ADX L345.

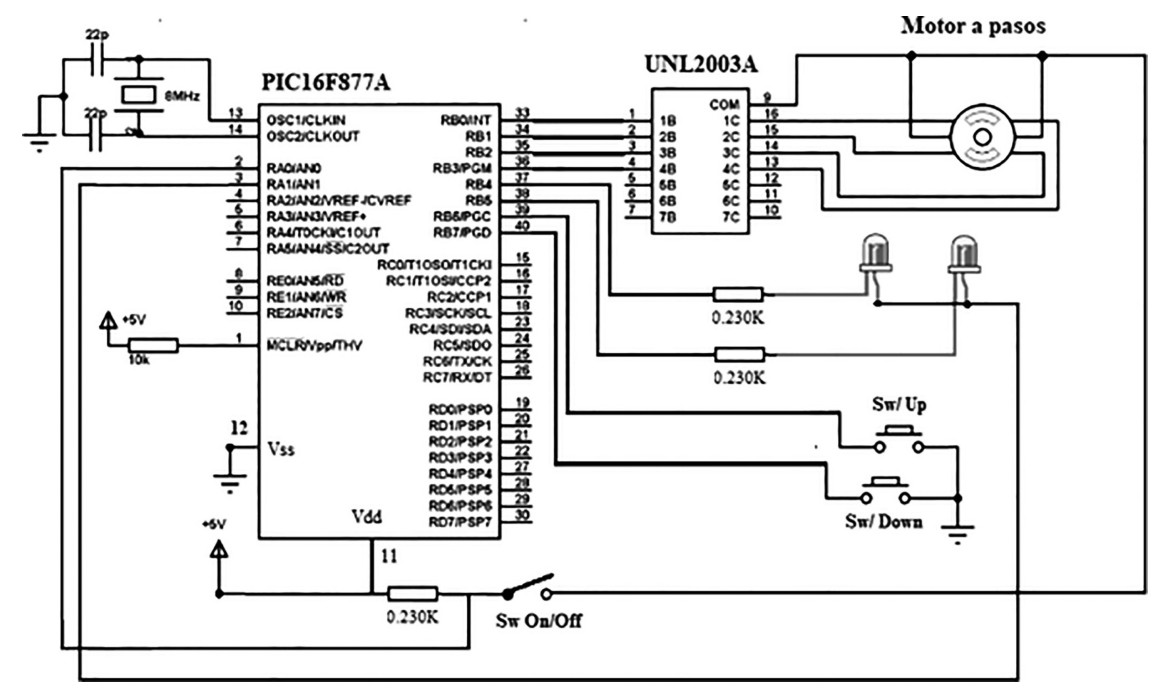

Figura 4: Diagrama de conexión del motor de pasos. Se puede identificar el controlador PIC 16F877A y el interruptor de control.

comienza a moverse (deja de ser constante la posición) y proyectar valor del ángulo para el cual ocurre (Figura 5. Esto permite descartar como fuente de error a cualquier efecto de tiempo de reacción o movimiento del plano luego de alcanzado el ángulo crítico.

\section{Determinación de coeficientes de fricción}

Con el fin de demostrar la utilidad del sistema propuesto, éste se utilizó para determinar el coeficiente de fricción entre diferentes tipos de superficies y la superficie de acrílico del plano inclinado. Para tal fin se utilizó un prisma rectangular fabricado en madera contra enchapada con dos caras cubiertas con recubrimientos de uso habitual para madera (Fórmica), de dimensiones $40 \mathrm{~mm} \times 40 \mathrm{~mm} \times 50 \mathrm{~mm}$. El prisma fue cortado de forma que todas las caras a estudiar tuvieran la misma superficie $\left(40 \times 50 \mathrm{~mm}^{2}\right)$. Las cuatro caras se definieron como: madera longitudinal, madera homogénea, fórmica rugosa y fórmica lisa. Los términos longitudinal y homogénea corresponden con la orientación de las capas contra enchapadas del prisma de 


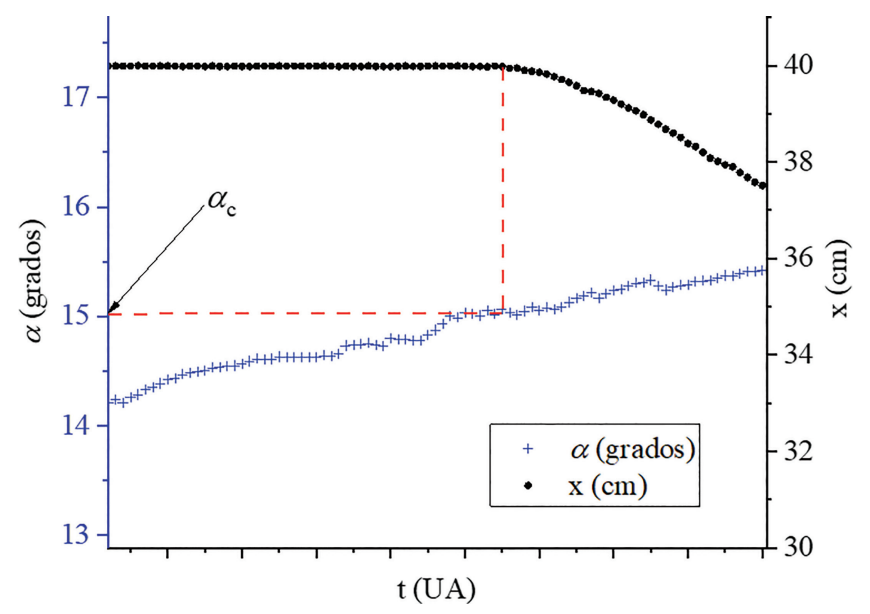

Figura 5: Medida de ángulo crítico a partir de la información de los sensores. La línea de puntos negros corresponde a la posición del móvil relativa al sensor de posición (x); cuando el movimiento es detectado se proyecta sobre la curva del ángulo del plano ( $\alpha$, cruces azules) y se interpola el ángulo crítico $\left(\alpha_{c}\right)$.

madera respecto al movimiento del cuerpo (Figuras 6 y 6f) y los términos rugosa y lisa corresponden al tipo de acabado del recubrimiento. En la Figura 6 se pueden apreciar imágenes de las 4 superficies estudiadas.

La determinación del coeficiente de fricción para cada cara fue realizada siguiendo el procedimiento antes descrito, cuidando de limpiar bien las superficies en contacto en cada medida, realizando 10 repeticiones para cada superficie, bajo condiciones idénticas.

Gracias a que el proceso de medición como tal no requiere de intervención humana directa, se pudo utilizar el sistema para evaluar el efecto de vibraciones externas sobre el coeficiente de fricción con una de las superficies señaladas anteriormente, la cara definida como "madera longitudinal".

Para evaluar el efecto de las vibraciones, el dispositivo se colocó en contacto solidario con un parlante de dimensiones similares a las de éste, todo el arreglo fue colocado sobre una mesa sencilla de madera. El parlante fue conectado a un generador de funciones, cuya la

frecuencia fue monitoreada con un osciloscopio. Se midió el coeficiente de fricción, siguiendo el procedimiento antes descrito, sometiendo el dispositivo a frecuencias $(F)$ entre $50 \mathrm{~Hz}$ y $600 \mathrm{~Hz}$, manteniendo constante la amplitud de la salida del parlante.

\section{Resultados y Discusión}

La Tabla 1 presenta el valor del coeficiente de fricción obtenido para cada superficie, a partir del valor medio obtenido para el ángulo crítico en cada caso y utilizando la ecuación (4).

Las incertidumbres obtenidas para los valores de coeficiente de fricción estática corresponden al error estadístico para las medidas, es decir provienen de la dispersión de los resultados obtenidos, estas dispersiones, por debajo del $2.8 \%$ en relación con la medida, demuestran un alto grado de reproducibilidad de las mediciones. Entre los resultados, el coeficiente con la menor incertidumbre $(0.3 \%)$, se obtuvo para la superficie denominada "madera longitudinal".

Establecer alguna correlación con valores reportados en la literatura, para verificar la exactitud de los resultados obtenidos, no es posible dadas las características que cada tipo de madera puede tener, además de que este coeficiente de fricción también se vería afectado por la rugosidad de los acabados de cada superficie, lo cual no está caracterizado. Aun así, los valores obtenidos para los coeficientes de fricción estática entre las superficies de madera y plástico son mayores a valores de coeficientes

Tabla 1: Coeficientes de fricción estática para las diferentes superficies estudiadas. Se observa como los recubrimientos artificiales tienen menor coeficiente de fricción estática.

\begin{tabular}{lc}
\hline Superficie & $\begin{array}{c}\text { Coeficiente de fricción } \\
\text { Estática }\left(\mu_{S}\right)\end{array}$ \\
\hline Madera longitudinal & $0.328 \pm 0.001$ \\
Madera Homogénea & $0.385 \pm 0.006$ \\
Fórmica rugosa & $0.317 \pm 0.003$ \\
Fórmica lisa & $0.289 \pm 0.008$ \\
\hline
\end{tabular}

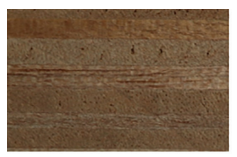

a

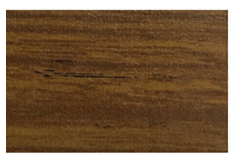

c

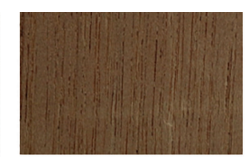

$b$

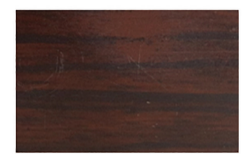

d e

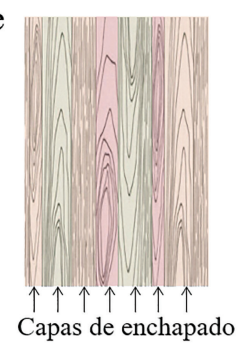

f

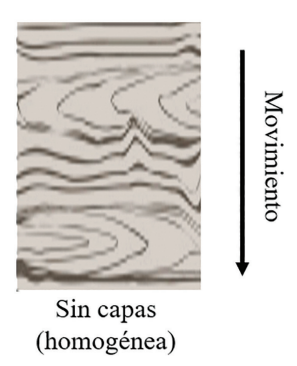

Figura 6: Superficies estudiadas. (a) madera longitudinal, (b) madera homogénea, (c) fórmica rugosa y (d) fórmica lisa. Un esquema de la disposición de las capas de madera natural se presenta en los insertos: (e) madera longitudinal, (f) madera homogénea. Las flechas indican la dirección de movimiento sobre el plano. 
de fricción cinética reportados en la literatura con precisiones similares a las reportadas en este trabajo (valores en torno a 0.2 ), lo cual es consecuente con la teoría [15, 16].

Se puede observar cómo los recubrimientos artificiales exhiben un menor coeficiente del fricción, notándose una marcada diferencia para la fórmica lisa, como era de esperar, estas son superficies mucho más lisas que las de la madera natural y por lo tanto el área real de contacto es mucho mayor, reduciendo la presión sobre cada punto.

Para evaluar el efecto de las vibraciones en el coeficiente de fricción se empleó la cara del prisma que mejores resultados produjo en cuanto a su reproducibilidad, la cara denominada "madera longitudinal". La Figura 7 muestra el gráfico de coeficiente de fricción en función de la frecuencia a la que fue sometido el sistema.

Se puede notar un leve efecto de las vibraciones sobre el coeficiente de fricción cuando se compara con los resultados presentados en la Tabla 1, aunque si se toma en cuenta la incertidumbre de las medidas, se puede considerar que no hay una relación directa entre estas dos variables, para la mayoría de las frecuencias estudiadas. Dentro de este comportamiento general, se observan en el gráfico (Figura 7) dos regiones donde el efecto de las vibraciones es mucho más marcado, una en torno a los $100 \mathrm{~Hz}$ y una en torno a los $400 \mathrm{~Hz}$, en ambos casos se observa cómo el coeficiente de fricción entre las superficies se reduce alrededor de un $10 \%$; se observó que estos valores de frecuencia correspondían a las respuestas resonantes de la mesa de trabajo y del equipo de mediciones en sí, por lo que estos valles en el gráfico corresponderían a las regiones en donde el montaje experimental era sometido a una mayor amplitud de oscilación, esto ocasionaría que la fuerza de

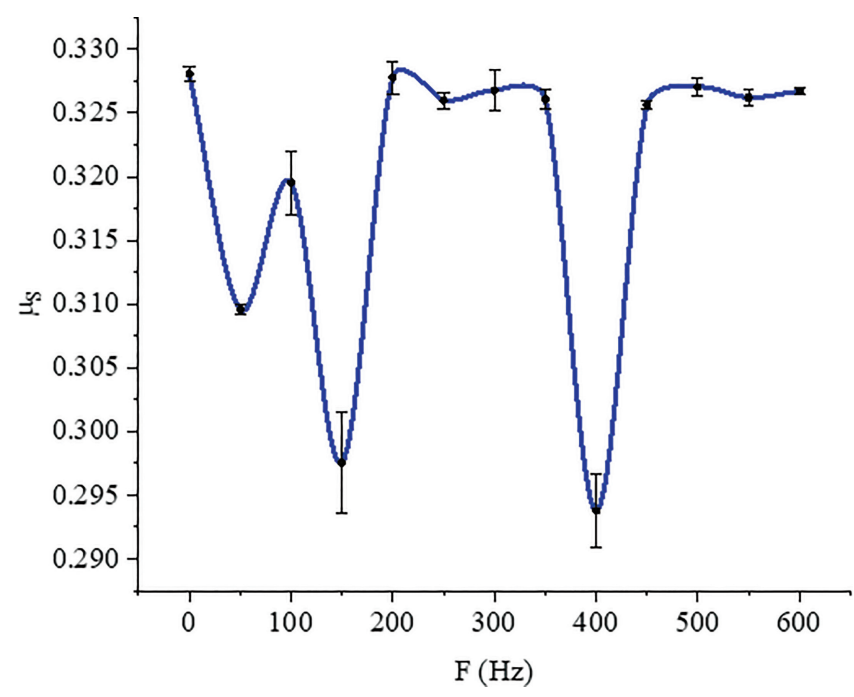

Figura 7: Coeficiente de fricción estática $\left(\mu_{s}\right)$ para la madera longitudinal, en función de la frecuencia de vibración a la que es sometido el sistema $(F)$. Se observan 2 valles pronunciados que se podrían atribuir a frecuencias de resonancia del montaje experimental. contacto entre las superficies se reduzca por el efecto de la inercia de los cuerpos, conllevando una reducción en la fuerza de fricción. Ya que este efecto no está tomado en cuenta en el modelo presentado normalmente a nivel universitario, se interpreta como un efecto directo sobre el coeficiente de fricción [17].

\section{Conclusiones y Consideraciones Finales}

El dispositivo presentado, al reducir la intervención humana en el momento de la medida, permite realizar mediciones muy reproducibles del coeficiente de fricción estática; la integración de los sensores de ángulo y posición para detectar el movimiento del cuerpo de pruebas, el control del movimiento del plano por medio de un motor, y el aislamiento mecánico de la superficie de mediada (para reducir vibraciones), permite obtener errores, asociados a dispersión estadística, inferiores al $3 \%$.

El equipo de medidas, orientado a prácticas de laboratorio para docencia, está construido con materiales sencillos y de fácil adquisición, por lo que su costo es relativamente bajo, esto permitiría la producción de múltiples unidades, por lo que se puede integrar como una actividad adicional dentro de un curso de laboratorio.

El uso de sensores electrónicos para medición y digitalización de datos experimentales, sobre todo en actividades de mecánica, puede ser especialmente estimulante para los alumnos, llevándolos a proponer nuevos desarrollos en instrumentación o simplemente facilitando su contacto con los laboratorios.

\section{Referências}

[1] J. Güémez y M. Fiolhais, European Journal of Physics 34, 4 (2013).

[2] J.A. Molina-Bolívar y M.A. Cabrerizo-Vílchez, Physics Education 49, 4 (2014).

[3] U. Besson, L. Borghi y A. De Ambrosis, American Journal of Physics 75, 12 (2007).

[4] U. Sari, Physics Education 54, 3 (2019).

[5] M.A. Kurnaz y C. Eksi, Educational Sciences: Theory \& Practice 15, 3 (2015).

[6] E.D. Corpuz y N.S. Rebello, Physical Review Special Topics - Physics Education Research 7, 2 (2011).

[7] T.J. Folkerts, American Journal of Physics 72, 10 (2004).

[8] A. Manzur, Revista Mexicana de Física E 54, 1 (2008).

[9] R. Serway y J. Jewett, Física para Ciencias e Ingeniería (Cengage Learning, Ciudad de México, 2008), v. 1.

[10] M. Kinsler y E. Kinzel, The Physics Teacher 44, 2 (2006).

[11] W.P. Castro, J.F. Arroyave y S. Acevedo, Scientia et technica 1, 44 (2010).

[12] J. Sobota, R. Pisl, P. Balda y M. Schlegel, IFAC Proceedings Volumes 46, 7 (2013). 
[13] P.H. Guadagnini, F.S. Rocha y V.E. Barlette, Revista Brasileira de Ensino de Física 41, 3 (2019).

[14] Š. Kubínová y J. Šlégr, Physics Education 50, 4 (2015).

[15] L.M. Gratton y S. Defrancesco, Physics Education 41, 3 (2006).

[16] Y. Murase, Journal of the Faculty of Agriculture, Kyushu University 28, 4 (1984).

[17] T. Majewskia y D. Szwedowiczb, en XXV Congreso Internacional Anual de la Somim (Sinaloa, México, 2019). 